\title{
DECOMPOSING ALGEBRAIC VECTOR BUNDLES ON THE PROJECTIVE LINE
}

\author{
CHARLES C. HANNA ${ }^{1}$
}

\begin{abstract}
T}(R)$ denotes the category of algebraic vector bundles on $\mathbf{P}_{R}^{1}, R$ a commutative, noetherian ring. If $K$ is a field, it is known that any $\mathscr{F} \in \mathcal{V}(K)$ is isomorphic to a (unique) direct sum of line bundles. If $\mathfrak{p} \in \operatorname{Spec} R$ and $K(\mathfrak{p})$ is the quotient field of $R / \mathfrak{p}$, any $\mathscr{F} \in \mathcal{V}(R)$ induces a bundle in $\checkmark(K(\mathfrak{p}))$, and so a decomposition into line bundles. If the decomposition is the same for each $\mathfrak{p}, \mathscr{F}$ is said to be uniform. It is shown that if $R$ is reduced, uniform vector bundles on $\mathbf{P}_{R}^{1}$ are sums of tensor products of (pullbacks of) bundles on $\operatorname{Spec} R$ with line bundles on $\mathbf{P}_{R}^{l}$.
\end{abstract}

Let $R$ be a commutative, noetherian ring with unit. $\mathscr{V}(R)$ denotes the category of algebraic vector bundles on the projective line over $R$. In [7], Roberts constructs indecomposable vector bundles of arbitrarily large rank. With a few hypotheses on $R$, our Theorem 7 shows that Roberts' construction is the only nontrivial way to obtain indecomposable vector bundles. We show that uniform bundles (bundles without the essential feature of Roberts' examples) are isomorphic to direct sums of constant bundles. A constant bundle is the tensor product of the pullback of a bundle on Spec $R$ with a line bundle on $\mathbf{P}_{R}^{1}$. The decomposability of constant bundles is really a question about $\mathscr{P}(R)$, the category of finitely generated projective $R$-modules.

1. Preliminaries. Following Bass [1, pp. 677-685], we think of $\mathscr{V}(R)$ in this way: The objects of $\Upsilon(R)$ are triples $\left(P_{+}, f, P_{-}\right)$, where $P_{+} \in \mathcal{P}(R[x]), P_{-}$ $\in \mathscr{P}(R[1 / x])$, and

$$
f: P_{+} \otimes_{R[x]} R[x, 1 / x] \rightarrow P_{-} \otimes_{R[1 / x]} R[x, 1 / x]
$$

is an $R[x, 1 / x]$-isomorphism. A morphism $\varphi:\left(P_{+}, f, P_{-}\right) \rightarrow\left(Q_{+}, g, Q_{-}\right)$is a pair of homomorphisms $\varphi_{ \pm}: P_{ \pm} \rightarrow Q_{ \pm}$with $\left(\varphi_{-} \otimes 1\right) \circ f=g \circ\left(\varphi_{+} \otimes 1\right)$.

If $\varepsilon: R \rightarrow T$ is a ring homomorphism and $\mathscr{F} \in \mathcal{V}(R)$, then $\mathscr{F} \otimes T$ is the pullback of $\mathscr{F}$ in $\mathscr{T}(T) ; S^{-1} \mathscr{F}$ is $\mathscr{F} \otimes S^{-1} R$ for any multiplicative set $S$ of $R$. If

Received by the editors February 10, 1976 and, in revised form, March 29, 1976.

AMS (MOS) subject classifications (1970). Primary 14F05, 13C10; Secondary 15A54, 18 G05.

Key words and phrases. Algebraic vector bundle, projective line, commutative noetherian ring, projective module, uniform vector bundle, decomposition of vector bundles.

1 Most of the results in this paper are contained in the author's thesis, written at the University of Chicago under the supervision of Professor Irving Kaplansky. The author would also like to thank Professor Leslie Roberts and the referee for their helpful suggestions. 
$P_{+}$and $P_{-}$are free of rank $n$, the bundle $\left(P_{+}, f, P_{-}\right)$is determined by an element of $\mathrm{GL}(n, R[x, 1 / x])$. If $\mathscr{F}$ and $\mathcal{G}$ are two such vector bundles represented by matrices $A$ and $A^{\prime}$ respectively, then $\mathscr{F}$ and $\mathcal{G}$ are isomorphic if and only if there exist matrices $B \in \mathrm{GL}(n, R[1 / x])$ and $C \in \mathrm{GL}(n, R[x])$ with $B A=A^{\prime} C$. We write $A \sim A^{\prime}$ in this case.

In [3] (cf. [2, Section 1], as well), Grothendieck proved that every vector bundle on $\mathbf{P}_{K}^{l}, K$ a field, is isomorphic to a direct sum of $O(n)$ 's, so that

$$
\mathscr{F} \cong \bigoplus_{i} r_{i} \mathcal{O}\left(n_{i}\right)
$$

The integers $r_{i}$ and $n_{i}$ are uniquely determined by $\mathscr{F}$. If $R$ is any ring, and $\mathfrak{p} \in \operatorname{Spec} R$, for $\mathscr{F} \in \mathcal{V}(R)$, we have a decomposition as above for $\mathscr{F} \otimes K(\mathfrak{p})$. This will be called the decomposition at $\mathfrak{p}$. If $\mathscr{F}$ has the same decomposition at all $\mathfrak{p} \in \operatorname{Spec} R$ we say $\mathscr{F}$ is uniform. (Of course, we may and do assume $\operatorname{Spec} R$ is connected.)

Observe that bundles which are pullbacks of bundles on $\operatorname{Spec} R$ tensored with $O(n)$ over $\mathbf{P}_{R}^{1}$ are uniform. Such bundles are called constant bundles. We

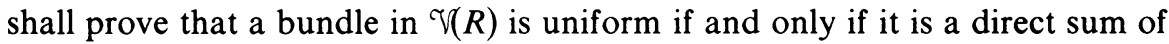
constant bundles, provided $R$ is reduced. This last hypothesis is necessary. Let $K$ be a field, $R=K[t] /\left(t^{2}\right)$. Let $\mathscr{F} \in \mathscr{V}(R)$ be represented by the matrix

$$
\left[\begin{array}{cc}
1 & 0 \\
t x & x^{2}
\end{array}\right]
$$

$\mathscr{F}$ is uniform since $R$ has only one prime, but it follows from Lemma 1 that $\mathscr{F}$ is not trivial.

2. The local case. Horrocks proved in [4] that if $R$ is a local ring, then bundles in $\checkmark(R)$ when restricted to $R[x]$ or $R[1 / x]$ are trivial. So when $R$ is local, any $\mathscr{F} \in \mathcal{V}(R)$ is represented by a matrix. Actually, Horrocks' proof (as in [6]) shows that the matrix $\left(a_{i j}\right)$ may be chosen so as to satisfy the following four conditions.

(1) If $i<j, a_{i j}=0$. (The matrix is lower triangular.)

(2) $a_{i i}=x^{e_{i}}$, with $e_{1} \leqslant \cdots \leqslant e_{n}$.

(3) If $i>j, a_{i j}$ is a polynomial in positive and negative powers of $x$. The degrees of the terms of $a_{i j}$ are all less than $e_{i}$ and greater than $e_{j}$. (So $a_{i j}=0$ if $e_{i}-e_{j} \leqslant 1$.)

(4) The decomposition of $\mathscr{F}$ at the maximal ideal of $R$ is $\Theta\left(-e_{1}\right) \oplus \cdots$ $\oplus \Theta\left(-e_{n}\right)$. (So if $K$ is the residue field of $R, \mathscr{F} \otimes K$ is represented by the diagonal matrix with entries $a_{i i}$.)

If $R$ is any ring and $\mathfrak{p} \in \operatorname{Spec} R$, for $\mathscr{F} \in \mathcal{V}(R), \mathscr{F} \otimes R_{\mathfrak{p}}$ may be represented by a matrix satisfying the above conditions. This is a triangular form at $\mathfrak{p}$, and is not unique in general. We say any matrix satisfying (1)-(3) is in triangular form. 
Lemma 1. Let $A, B \in \mathrm{GL}(n, R[x, 1 / x])$. Suppose that $A$ is a diagonal matrix, that $B$ is in triangular form, and that the diagonal entries of $B$ are the same as those of $A$. If $A \sim B$, then $A=B$.

The proof is a computation, and will be omitted.

If $A=\left(a_{i j}\right) \in \mathrm{GL}(m, R[x, 1 / x])$ is in triangular form, let $I(A)$ denote the ideal of $R$ generated by all the coefficients of all the polynomials $a_{i j}, i>j$. In particular, $I(A)=0$ means $A$ is diagonal.

Corollary 2. Let $R$ be local with maximal ideal $\mathrm{m}$, and let $\mathscr{F} \in \mathcal{V}(R)$. If $A$ is a triangular form for $\mathscr{F}$ at $\mathrm{m}$, then $I(A) \subset \mathrm{m}$.

Proof. Let $\varepsilon: R \rightarrow R / \mathrm{m}$ be the canonical map, and let $B$ be the matrix obtained by applying $\varepsilon$ to the coefficients of the entries of $A$. Then $B$ represents $\mathscr{F} \otimes R / \mathrm{m}$, and Grothendieck's theorem says there is a diagonal matrix $C$ with $B \sim C$. Horrocks' theorem implies that $B$ and $C$ have the same diagonal elements, so $B=C$ by Lemma 1 . Since $I(B)=0, I(A) \subset \mathrm{m}$.

Lemma 3. Let $R$ be local and reduced. Then any uniform bundle in $\mathcal{V}(R)$ is isomorphic to a direct sum of constant bundles (and so to a direct sum of $\Theta(n)$ 's).

Proof. Let $\mathscr{F}$ be uniform, and $A$ a triangular form for $\mathscr{F}$ at the maximal ideal of $R$. It suffices to show that $A$ is diagonal. For each $\mathfrak{p} \in \operatorname{Spec} R$, let $A_{\mathfrak{p}} \in \mathrm{GL}\left(n, R_{\mathfrak{p}}[x, 1 / x]\right)$ be the matrix induced by $A$. Then $A_{\mathfrak{p}}$ is a triangular form for $\mathscr{F} \otimes R_{\mathfrak{p}}$ at $\mathfrak{p} R_{\mathfrak{p}}$, so $I(A)_{\mathfrak{p}}=I\left(A_{\mathfrak{p}}\right) \subset \mathfrak{p} R_{\mathfrak{p}}$. This implies $I(A) \subset \mathfrak{p}$, for all $\mathfrak{p}$, and so $I(A)=0$ as $R$ is reduced.

Lemma 4. Suppose that $R$ is reduced. Then $\mathscr{F} \in \mathcal{V}(R)$ is uniform if and only if the triangular forms for $\mathcal{F}$ at each $\mathfrak{p} \in \operatorname{Spec} R$ are diagonal matrices.

Proof. Necessity is an immediate consequence of Lemma 3. To show sufficiency, let $\mathfrak{p} \subset \mathfrak{q}$ be prime ideals of $R$. Let $A$ and $B$ be the diagonal matrices representing $\mathscr{F} \otimes R_{\mathrm{p}}$ and $\mathscr{F} \otimes R_{\mathrm{a}}$, respectively. The localization $R_{\mathrm{a}}$ $\rightarrow R_{\mathfrak{p}}$ induces a map GL $\left(n, R_{\mathrm{q}}[x, 1 / x]\right) \rightarrow \mathrm{GL}\left(n, R_{\mathfrak{p}}[x, 1 / x]\right)$ which must take $A$ to $B$. (The uniqueness in Grothendieck's theorem is valid for any ring.) So $\mathscr{F}$ has the same decomposition at $\mathfrak{p}$ as at $\mathfrak{a}$, and the result follows from the connectedness of $\operatorname{Spec} R$.

3. Cohomology of uniform bundles. Following Serre [8, Section 54] we write $\mathscr{F}(n)$ for $\mathscr{F} \otimes \mathcal{O}(n)$.

LEMMA 5. Let $R$ be reduced. $\mathscr{F} \in \mathcal{V}(R)$ is uniform if and only if for each integer $n, H^{i}(\mathscr{F}(n))$ is a projective $R$-module. ( $i=0$ or 1 ; higher cohomology is always 0.)

Proof. Suppose $\mathscr{F}$ is uniform and $\mathfrak{p} \in \operatorname{Spec} R$. Then

$$
H^{i}(\mathscr{F}(n))_{\mathfrak{p}} \cong H^{i}\left(\mathscr{F}(n) \otimes R_{\mathfrak{p}}\right)
$$


$\mathscr{F}(n) \otimes R_{\mathfrak{p}}$ is a direct sum of $\Theta(n)$ 's by Lemma 3, and $H^{i}(\mathcal{O}(n))$ is free. Since $R$ is noetherian, $H^{i}(\mathscr{F}(n))$ is finitely generated, and so projective.

Conversely, suppose $\mathscr{F}$ has different decompositions at two primes. Since $H^{1}(\mathscr{F}(n)) \otimes_{R} K(\mathfrak{p}) \cong H^{1}(\mathscr{F}(n) \otimes K(\mathfrak{p}))$ for all $\mathfrak{p} \in$ Spec $R$, there is some $n$ for which $H^{1}(\mathscr{F}(n))$ has different ranks at the two primes. If $H^{1}(\mathscr{F}(n))$ were projective, this would contradict the connectedness of $\operatorname{Spec} R$.

For $\mathscr{F}=\left(P_{+}, f, P_{-}\right)$, we identify $H^{0}(\mathscr{F}(n))$ with

$$
\left\{(p, q) \in P_{+} \otimes_{R} P_{-}: f(p \otimes 1)=x^{n}(q \otimes 1)\right\} .
$$

Let $m \leqslant n$ be integers and $r=n-m$. Let $M$ be a direct sum of $r+1$ copies of $H^{0}(\mathscr{F}(m))$, and define $\alpha_{m, n}: M \rightarrow H^{0}(\mathscr{F}(n))$ by

$$
\begin{aligned}
\alpha_{m, n}\left(\left(p_{0}, q_{0}\right),\left(p_{1}, q_{1}\right)\right. & \left.\ldots,\left(p_{r}, q_{r}\right)\right) \\
& =\left(x^{r} p_{0}+x^{r-1} p_{1}+\cdots+p_{r}, q_{0}+x^{-1} q_{1}+\cdots+x^{-r} q_{r}\right) .
\end{aligned}
$$

LEMMA 6. If $\mathscr{F} \in \mathfrak{V}(R)$ is uniform, the image of each $\alpha_{m, n}$ is a direct summand of $H^{0}(\mathscr{F}(n))$.

Proof. We need only prove the result locally. Since localization at a multiplicative set in $R$ commutes with the formation of $H^{0}(\mathscr{F}(n))$, we may assume that $R$ is a local ring. But in this case $\mathscr{F}$ is a direct sum of $\theta(n)$ 's, so represented by a diagonal matrix. The lemma then follows routinely.

\section{The main theorem.}

THEOREM 7. Let $R$ be a commutative, noetherian ring with unit, reduced and with connected prime spectrum. Then $\mathscr{F} \in \mathcal{V}(R)$ is uniform if and only if it is isomorphic to a direct sum of constant bundles.

Proof. Only one implication requires proof. Assume that $\mathscr{F}=\left(P_{+}, f, P_{-}\right)$is uniform, with decomposition at each prime $\oplus_{1 \leqslant i \leqslant t} r_{i} \mathcal{O}\left(-n_{i}\right)$ where $n_{1}<\cdots$ $<n_{t}$. We will construct $t$ constant bundles and an isomorphism from their direct sum to $\mathscr{F}$.

Let $P_{1}=H^{0}\left(\mathscr{F}\left(n_{1}\right)\right)$. For $2 \leqslant i \leqslant t$, let $P_{i}$ be any $R$-submodule of $H^{0}\left(\mathscr{F}\left(n_{1}\right)\right)$ such that $H^{0}\left(\mathscr{F}\left(n_{1}\right)\right)$ is a direct sum of $P_{i}$ and the image of $\alpha_{i}\left(=\alpha_{m, n}\right.$ as defined above, with $m=n_{i-1}$ and $\left.n=n_{i}\right)$. For $1 \leqslant i \leqslant t, P_{i}$ is a projective $R$-module of rank $r_{i}$.

Define $\mathcal{G}_{i}=\left(P_{i} \otimes_{R} R[x], 1 \otimes x^{n_{i}}, P_{i} \otimes_{R} R[1 / x]\right)$, a constant bundle. Let $\mathcal{G}$ $=\mathcal{G}_{1} \oplus \cdots \oplus \mathcal{G}_{t}$, a bundle of the same rank as $\mathscr{F}$. If $\mathcal{G}=\left(Q_{+}, g, Q_{-}\right)$, elements of $Q_{+}$are sums of terms of the form $a=(p, q) \otimes r$, where $(p, q) \in H^{0}\left(\mathscr{F}\left(n_{i}\right)\right)$ for some $i$ and $r \in R[x]$. Set $\varphi_{+}(a)=r p \in P_{+} ; \varphi_{-}: Q_{-} \rightarrow P_{-}$is defined similarly. It is easy to verify that we have a morphism $\varphi: \mathcal{G} \rightarrow \mathscr{F}_{\text {. }}$

In fact, $\varphi$ is an isomorphism, i.e., $\varphi_{+}$and $\varphi_{-}$are isomorphisms. To show this, we need only establish that $\varphi_{+}$and $\varphi_{-}$are surjective, since their domains and codomains are projectives of the same (finite) rank. We will prove that $\varphi_{+}$is 
surjective; the argument for $\varphi_{-}$is the same.

We claim that if $p \in P_{+}$satisfies

$$
\text { for some } q \in P_{-} \text {and some } i,(p, q) \in H^{0}\left(\mathscr{F}\left(n_{i}\right)\right) \text {, }
$$

then $p$ is in the image of $\varphi_{+}$. If $i=1$, this is obvious; otherwise, write $(p, q)$ as the sum of an element of $P_{i}$ and something in the image of $\alpha_{i}$. This yields an expression for $p$ as a linear combination (over $R[x]$ ) of elements $p_{j}$, where for some $q_{j},\left(p_{j}, q_{j}\right)$ is either in $P_{i}$ or in $H^{0}\left(\mathscr{F}\left(n_{i-1}\right)\right)$. Induction on $i$ then establishes the claim.

When $R$ is local, $P_{+}$is generated by elements satisfying $(*)$, so $\varphi_{+}$is surjective. In the general case we need only show that $S^{-1} \varphi: S^{-1} Q_{+} \rightarrow S^{-1} P_{+}$ is surjective whenever $S$ is the complement in $R[x]$ of some $\mathfrak{p} \in \operatorname{Spec} R[x]$. But it suffices to localize with respect to $S \cap R$, which amounts to assuming that $R$ is local. This completes the proof.

(Note that only the easier implications of Lemmas 4 and 5 were used in the proof of Theorem 7.)

If $R$ is as in Theorem 7 and $\mathscr{F}=\left(P_{+}, f, P_{-}\right) \in \mathcal{V}(R)$ is uniform, it is an immediate corollary that $P_{+}$is extended from some $P \in \mathscr{P}(R)$. Quillen has recently proved in [6] that this is true for arbitrary $\mathscr{F} \in \mathcal{V}(R), R$ any commutative ring with unit. He uses this result to answer Serre's question in the affirmative.

\section{REFERENCES}

1. H. Bass, Algebraic K-theory, Benjamin, New York, 1968. MR 40 \#2736.

2. G. D. Birkhoff, Singular points of ordinary linear differential equations, Trans. Amer. Math. Soc. 10 (1909), 436-470.

3. A. Grothendieck, Sur la classification des fibres holomorphes sur la sphere de Riemann, Amer. J. Math. 79 (1957), 121-138. MR 19, 315.

4. G. Horrocks, Projective modules over an extension of a local ring, Proc. London Math. Soc. (3) 14 (1964), 714-718. MR 30 \#121.

5. M. P. Murthy, Projective modules, Lecture Notes, Univ. of Chicago (to appear).

6. D. Quillen, Projective modules over polynomial rings (to appear).

7. L. Roberts, Indecomposable vector bundles on the projective line, Canad. J. Math. 24 (1972), 149-154. MR 45 \#6828.

8. J.-P. Serre, Faisceaux algebriques coherents, Ann. of Math. (2) 61 (1955), 197-278. MR 16, 953.

Department of Mathematics, Louisiana State University, Baton Rouge, Louisiana 70803 